\title{
Nanoparticle impacts reveal magnetic field induced agglomeration and reduced dissolution rates
}

\author{
Kristina Tschulik $^{* a}$ and Richard G Compton ${ }^{* a}$ \\ Received (in $X X X, X X X)$ Xth $X X X X X X X X X 20 X X$, Accepted Xth $X X X X X X X X X 20 X X$ \\ DOI: 10.1039/b000000x
}

\begin{abstract}
Superparamagnetic nanoparticles (NPs) are used in a variety of magnetic field-assisted chemical and medical applications, yet little of their fate during magnetic field interrogation is known. Here, fundamental and new insights in this are gained by cathodic particle coulometry. This methodology is used to study individual $\mathrm{Fe}_{3} \mathrm{O}_{4} \mathrm{NPs}$ in the presence and absence of a magnetic field. It is first noticed that 10 no major NP agglomeration occurs in the absence of a magnetic field even in a suspension of high ionic strength. In contrast, a significant magnetic field-induced agglomeration of NPs is observed in a magnetic field. A second new finding is that the dissolution of $\mathrm{Fe}_{3} \mathrm{O}_{4} \mathrm{NPs}$ is strongly inhibited in a magnetic field. This is explained as a result of the magnetic field gradient force trapping the released $\mathrm{Fe}^{2+}$ ions near the surface of a magnetized $\mathrm{Fe}_{3} \mathrm{O}_{4} \mathrm{NP}$ and thus hindering the mass-transport controlled NP dissolution.

${ }_{15}$ Consequently, fundamental magnetic field effects are measured and quantified on the single NP scale and in suspension and two novel effects are discovered.
\end{abstract}

\section{Introduction}

Superparamagnetic magnetite nanoparticles $\left(\mathrm{Fe}_{3} \mathrm{O}_{4} \mathrm{NPs}\right)$ find widespread applications in catalysis and biotechnology for 20 magnetic field assisted separation and recovery. ${ }^{1}$ They are also frequently used in standard biomedical applications, the most prominent example being contrasting agents in magnetic resonance imaging (MRI). ${ }^{2}$ Recent pre-clinical studies additionally outlined the potential of $\mathrm{Fe}_{3} \mathrm{O}_{4}$ NPs for cancer 25 treatment by locally induced cell apoptosis, either by magnetic field-induced local heating (hyperthermia) or magnetic field directed drug delivery. ${ }^{3}$ Despite often being considered as biocompatible, numerous studies show that the toxicity of iron oxide nanoparticle varies significantly with particle concentration, 30 capping agent, size, size distribution and agglomeration state..$^{2,45}$ The effect of NP size, concentration and capping agent has been studied in much detail. Contrary, studies concerning the agglomeration state (and hence the size distribution), which strongly depends on the surrounding liquid, are rare and there is a 35 lack of investigations on the effects of applied magnetic fields on the agglomeration of $\mathrm{Fe}_{3} \mathrm{O}_{4} \mathrm{NPs}$ in suspension. The recently developed method of electrochemically sizing individual NPs by particle coulometry fills this gap, as will be shown in this work. This method uses the Brownian motion driven impacts of 40 suspended NPs at a microelectrode to reduce or oxidize individual NPs. The charge passed during an impact event is determined by the number of atoms in the NP and thus the NP size can be inferred. ${ }^{6}$

Herein, cathodic particle coulometry, this is the reductive ${ }_{45}$ dissolution of single $\mathrm{Fe}_{3} \mathrm{O}_{4}$ NPs, is used to study the agglomeration as well as the size distribution of these NPs in suspension and in the presence of a magnetic field. In contrast to common laser-based sizing techniques, where the application of magnetic fields would be a challenging task, it will be shown that 50 this analysis can conveniently be performed in a magnetic field. It is observed that even in the low magnetic field of a permanent magnet $(0.3 \mathrm{~T})$ significant agglomeration occurs and that the dissolution of $\mathrm{Fe}_{3} \mathrm{O}_{4}$ is remarkably retarded.

Very recently, Santos et al. ${ }^{7}$ used a similar electrochemical 55 approach to detect prussian blue coated $\mathrm{Fe}_{3} \mathrm{O}_{4}$ NPs upon oxidizing this capping agent during NP impact experiments. They showed that a magnetic field of $0.2 \mathrm{~T}$ can direct the nanoparticles away from the working electrode, which is detected by the disappearance of current spikes. The $\mathrm{Fe}_{3} \mathrm{O}_{4}$ concentration used in ${ }_{60}$ their study was in the order of $10^{-4} \mathrm{~g} / \mathrm{mL}$. At this concentration inter-particle interactions of the suspended superparamagnetic $\mathrm{Fe}_{3} \mathrm{O}_{4}$ nanoparticles are significant and they show a ferrofluidlike behaviour ${ }^{8}$. This associative character results in physical effects that are not usually observed for nanoparticulate systems. 65 such as sedimentation and, when subjected to a magnetic field, bulk-like agglomeration to one big agglomerate. Both these examples are among the interesting observations reported by Santos et al.

The concentration of $\mathrm{Fe}_{3} \mathrm{O}_{4}$ NPs used in the present work is in 70 the order of $10^{-8} \mathrm{~g} / \mathrm{mL}$, as we aim to study concentrations that correspond to those resulting during medical treatments. At this low concentration NP interactions are less dominant and the suspension characteristics closely reflect those of individual NPs. Studying these by cathodic particle coulometry yields novel 75 insights into the fate of magnetic NPs during magnetic field interrogation. Specifically, a magnetic field-induced agglomeration of individual NPs into small clusters and a strongly retarded dissolution of the magnetized NPs are observed. 


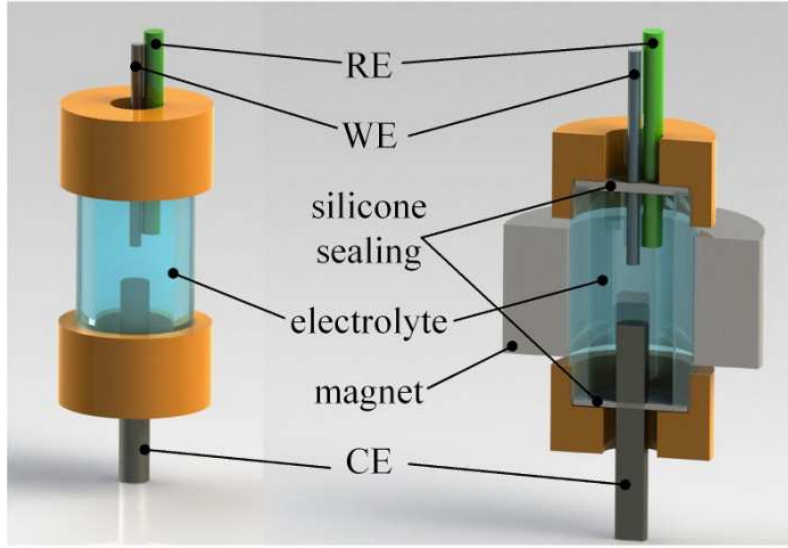

Fig. 1 Schematic drawing of the electrochemical setup. The cylindrical electrochemical cell is placed in a $\mathrm{NdFeB}$ ring magnet for experiments in the presence of a magnetic field as indicated in the right image; $\mathrm{WE}=$ 5 C fibre micro electrode, $\mathrm{CE}=$ graphite rod, $\mathrm{RE}=\mathrm{SCE}$, the cell body is made of PEEK (polyether ether ketone) and the electrolyte is a $0.2 \mathrm{M}$ phosphate buffer solution ( $\mathrm{pH} 10)$.

\section{Experimental}

Nanoparticle impact experiments at a micro carbon fibre working 10 electrode (WE, $\varnothing=11 \mu \mathrm{m}$ ) are performed at room temperature in a three electrode setup, as sketched in Figure 1. A graphite rod $(\varnothing=3 \mathrm{~mm})$ serves as the counter electrode (CE) and a calomel electrode ( $\mathrm{SCE} ; \mathrm{Hg} / \mathrm{Hg}_{2} \mathrm{Cl}_{2}, \mathrm{KCl}_{\text {(sat) }}$, potential $E=0.24 \mathrm{~V}$ vs standard hydrogen electrode) is used as the reference electrode.
${ }_{15}$ Experiments are controlled by a $\mu$ Autolab II potentiostat (Metrohm-Autolab)

For measurements in the presence of a magnetic field, the cylindrical electrochemical cell is placed in a neodymium iron boron $(\mathrm{NdFeB})$ ring permanent magnet (outer $\varnothing=24 \mathrm{~mm}$, inner ${ }_{20} \varnothing=12 \mathrm{~mm}, h=8 \mathrm{~mm}$; maximum energy product $=42 \mathrm{MOe}$ ), as visualized in Figure 1. The magnetic field at the position of the working electrode is $0.3 \mathrm{~T}$, as shown in section S1 of the SI.

A $0.2 \mathrm{M}$ phosphate buffer solution of $\mathrm{pH} 10$ is prepared by dissolving $0.001 \mathrm{M} \mathrm{K}_{3}\left(\mathrm{PO}_{4}\right)$ (potassium phosphate tribasic) and ${ }_{25} 0.199 \mathrm{M} \mathrm{K}_{2} \mathrm{H}\left(\mathrm{PO}_{4}\right)$ (potassium phosphate dibasic) in de-ionised water (Millipore, resistivity at $25^{\circ} \mathrm{C}$ not less than $18.2 \mathrm{M} \Omega \mathrm{cm}$ ). After addition of $40 \mu \mathrm{L}$ of a suspension of uncapped commercial $\mathrm{Fe}_{3} \mathrm{O}_{4} \mathrm{NP}\left(1.5 \mathrm{mg}\right.$ in $1 \mathrm{~mL} \mathrm{H} \mathrm{H}_{2} \mathrm{O}$; mean radius $=12 \pm 4 \mathrm{~nm}$ (see $\mathrm{SI})$ ) to $2 \mathrm{~mL}$ of this electrolyte the suspension (now containing 30 $30 \mathrm{ng} \mathrm{Fe}_{3} \mathrm{O}_{4}$ per $\mathrm{mL}$ ) is first sonicated for $30 \mathrm{~s}$ and then deaerated by purging $\mathrm{N}_{2(\mathrm{~g})}$ for 15 minutes prior to the experiments. All chemicals were purchased in analytical grade from Sigma Aldrich, UK.

Two series of chronoamperograms were recorded with and 35 without an applied magnetic field at a potential of $-0.9 \mathrm{~V}$ vs SCE for $5 \mathrm{~s}$, to obtain a statistical relevant number of reductive particle impacts for both setups. As shown elsewhere ${ }^{9}$, this potential ensures quantitative reduction and thus sizing of $\mathrm{Fe}_{3} \mathrm{O}_{4} \mathrm{NP}$ by cathodic particle coloumetry. Both sets of experiments were 40 analyzed with respect to the duration and the charge transferred for each of the detected individual current spikes.
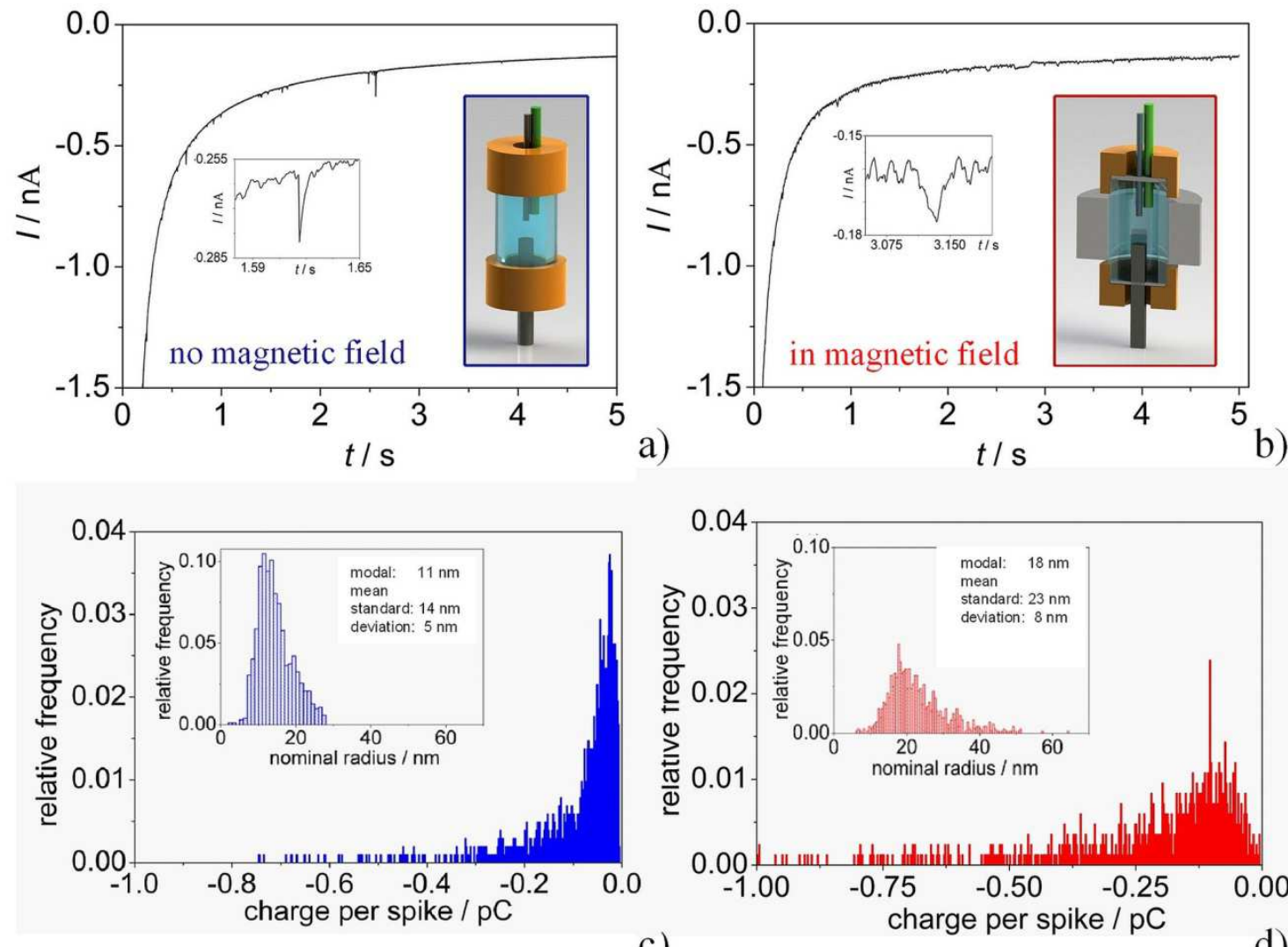

c)

d)

Fig. 2 Chronoamperograms showing reductive impact spikes recorded without (a) and in (b) a magnetic field and the derived impact charge and NP size 45 distributions (c,d); electrolyte: $0.2 \mathrm{M}$ phosphate buffer $(\mathrm{pH}=10), \mathrm{E}_{\mathrm{SCE}}=-0.9 \mathrm{~V}$. 


\section{Cite this: DOI: $10.1039 / \mathrm{c0xx00000x}$}
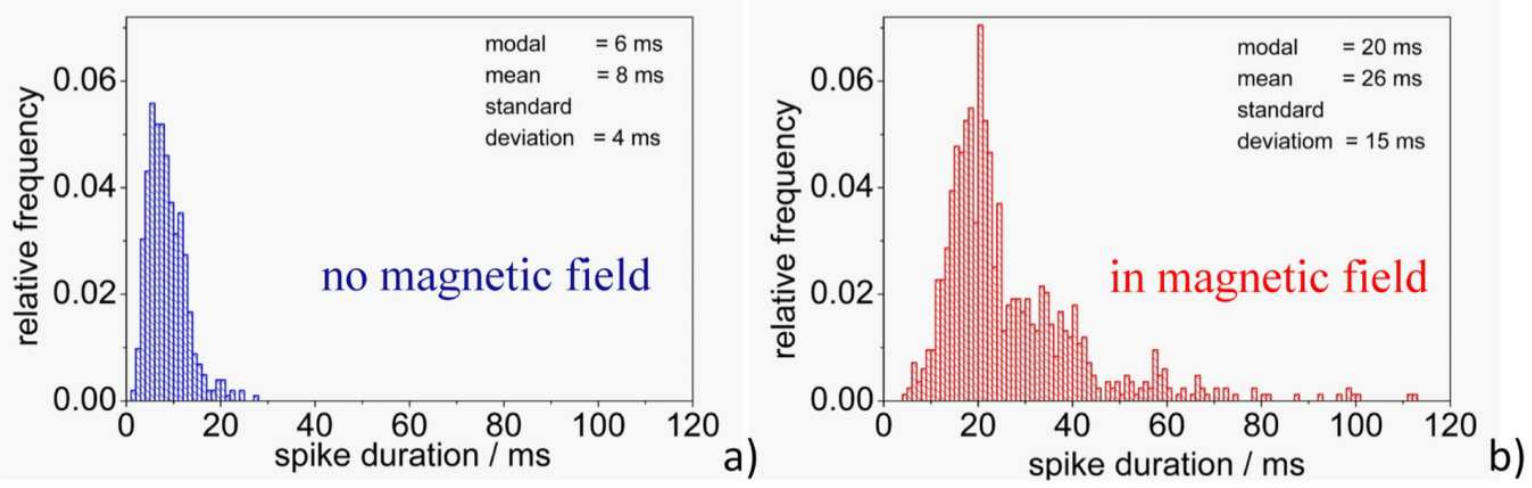

Fig. 3 Distribution of the durations of impact spikes (a) in the absence and (b) in the presence of a magnetic field of 0.3 T; electrolyte: $0.2 \mathrm{M}$ phosphate buffer $(\mathrm{pH}=10), E_{\mathrm{SCE}}=-0.9 \mathrm{~V}$.

\section{s 3 Results and discussion}

Characteristic chronoamperograms recorded during the abovementioned impact experiments are plotted in Figure 2. Characteristic current spikes are visible in both the absence (Figure 2a) and the presence of a magnetic field of $0.3 \mathrm{~T}$ (Figure $102 \mathrm{~b}$ ). These current spikes originate from the electrochemical reduction of $\mathrm{Fe}_{3} \mathrm{O}_{4}$ NPs impacting the electrode, thereby consuming two electrons per formula unit under formation of $\mathrm{Fe}^{2+}$. As reported previously, the reductive charge $(Q)$ associated with each spike allows for determination of the size of the 15 impacting particle according to: ${ }^{6}$

$$
r=\sqrt[3]{\frac{3 M Q}{4 \pi \mathrm{e} \mathrm{N}_{\mathrm{A}} z \rho}}
$$

where $r$ is the NP radius assuming a spherical NP shape, $M$ is the molar mass and $\rho$ is the density of $\mathrm{Fe}_{3} \mathrm{O}_{4}, z$ is the number of electrons exchanged per formula unit of $\mathrm{Fe}_{3} \mathrm{O}_{4}$ and $\mathrm{N}_{\mathrm{A}}$ is the 20 Avogadro constant.

In the absence of a magnetic field, the modal charge transferred per impact event is found to be $-0.025 \mathrm{pC}$ (Figure 2c). Using Eq. 1 this charge corresponds to a NP radius of about $11 \mathrm{~nm}$, which is the value measured for monomers during SEM 25 investigations (see Fig. S2 in the SI). This shows that the suspended $\mathrm{Fe}_{3} \mathrm{O}_{4}$ NPs negligibly agglomerate in the suspension despite the high ionic strength of the electrolyte.

In contrast, in the presence of a magnetic field of $0.3 \mathrm{~T}$ the modal charge increases to $-0.1 \mathrm{pC}$ (Figure 2d), which is four 30 times the value of a monomer particle, and a much wider distribution of impact charges is observed. Considering the superparamagnetic character of the used NPs this observation can be attributed to magnetization of the $\mathrm{Fe}_{3} \mathrm{O}_{4} \mathrm{NPs}$ in the external field, causing attractive forces between the NPs in suspension and 35 hence their agglomeration upon collision with each other. Thus,
NP impact experiments in an applied magnetic field revealed magnetic-field induced agglomeration and furthermore allowed quantifying the extent of this magnetic field-induced NP agglomeration in a NP suspension.

40 Consistent with the increased spike charges, the measured duration of individual impact spikes is much longer in the presence of a magnetic field (Figure 3). This observation agrees with the theoretical prediction that once a NP is sufficiently close to the electrode for charge transfer to occur, it most likely resides 45 at or close to the electrode until it is fully reduced. ${ }^{10}$

Thus, magnetic field-induced NP agglomeration increases the size of impacting NPs and this causes an increase in the observed spike duration. However, it is also known that the limiting current at a NP (acting as a nano-electrode) increases linearly with 50 increasing particle radius. ${ }^{11,12,{ }^{\dagger 1}}$ As a result, the peak duration for agglomerates should increase to a much lesser extent than observed experimentally.

To elucidate this finding in more detail, the measured charge per spike is plotted against its duration (up to $30 \mathrm{~ms}$ ) in the 55 absence (a) and presence (b) of an applied magnetic field in Figure 4 for individual NP impacts (a comparison of the full range of durations can be found in Fig. 3 in the SI). A general trend of increasing charge transferred per spike with increasing peak duration can be inferred for both. In a magnetic field this 60 correlation appears to be more pronounced and it is shifted to longer durations. In other words, in an applied magnetic field transferring the same charge between NP and electrode takes longer. This becomes more obvious when plotting the mean spike current transferred per time (this is the charge transferred per 

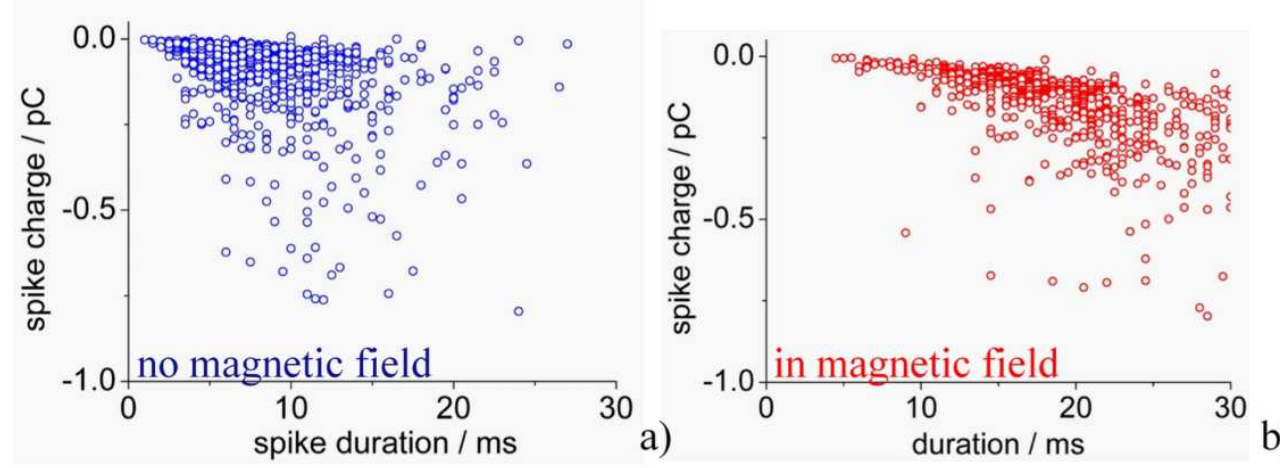

Fig. 4 Transferred charge per individual spike against its duration (a) without and (b) in a magnetic field of 0.3 T; electrolyte: $0.2 \mathrm{M}$ phosphate buffer $(\mathrm{pH}=10), E_{\mathrm{SCE}}=-0.9 \mathrm{~V}$.
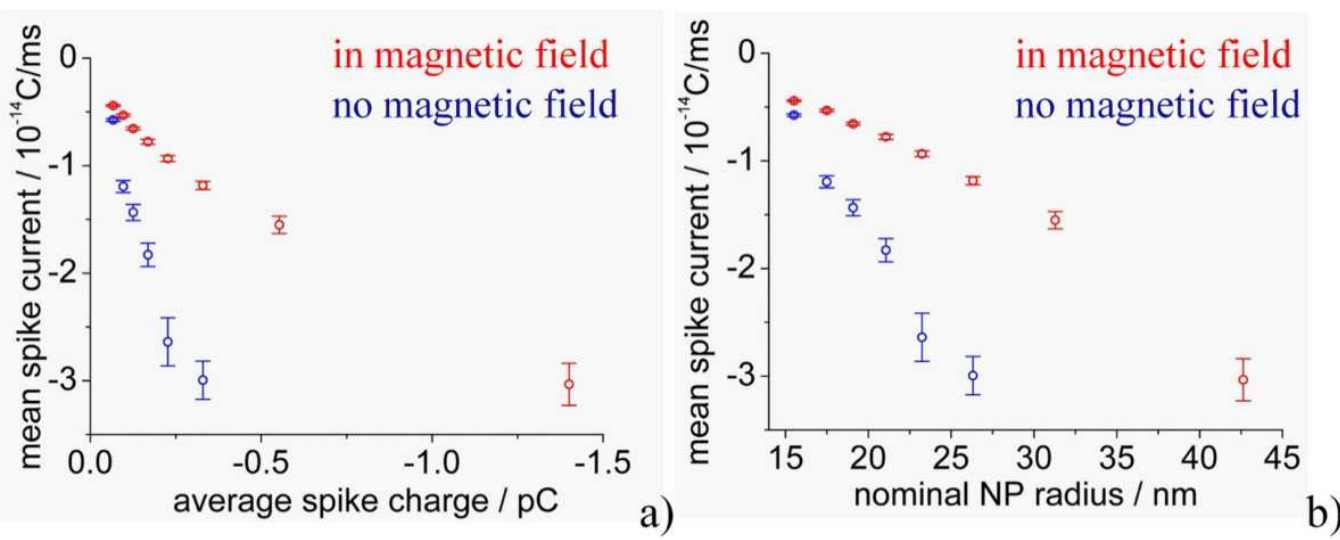

Fig. 5 Mean currents per impact spike in dependence of the average individual spike charge (a) and the corresponding nominal radius (b); electrolyte: $0.2 \mathrm{M}$ phosphate buffer $(\mathrm{pH}=10), E_{\mathrm{SCE}}=-0.9 \mathrm{~V}$.

individual spike divided by its duration) against the totally transferred charge for this individual spike.

10 In Figure 5 this relation is compared for impacts in presence and absence of a magnetic field. The fact that the mean current increases with increasing average spike charge simply reflects the radius dependency of the limiting current to or from a nanoparticle/-electrode mentioned earlier. ${ }^{13}$

${ }_{15}$ Using eq. 1 we can assign a nominal (hydrodynamic) NP radius to the detected peak area and the average charge per nominal NP radius indeed follows an almost linear trend (Figure 5b). Note that due to the fact that agglomerates of NPs likely differ from an ideal spherical shape and so a 20 detailed analysis of the mean current to NP size relation requires a further computational analysis.

However, it is evident that for all data points the mean current is significantly lower in an applied magnetic field than it is in absence of magnetic forces. As the strength of 25 magnetic fields generated by permanent magnets or magnetized NPs are (at least) three orders of magnitude too small to cause a significant shift in the thermodynamics ${ }^{14}$ or electrode kinetics, ${ }^{15}$ these changes in mean current must be caused by a hindered mass transport. This reduced mass 30 transport can be attributed to the action of the magnetic field gradient force, as will be discussed in the following paragraph.

Even in a small external magnetic field (here $0.3 \mathrm{~T}$ ) superparamagnetic $\mathrm{Fe}_{3} \mathrm{O}_{4} \mathrm{NPs}$ are magnetized and hence act 35 as nano-magnets themselves (the saturation magnetization of $\mathrm{Fe}_{3} \mathrm{O}_{4}=0.6 \mathrm{~T}^{16}$ ). As the magnetic permeability of $\mathrm{Fe}_{3} \mathrm{O}_{4}$ is three orders of magnitude bigger than that of the electrochemical system, ${ }^{16}$ this magnetization drops to zero in very close proximity to an $\mathrm{Fe}_{3} \mathrm{O}_{4} \mathrm{NP}$, causing a localized 40 strong magnetic gradient directly at the NP surface. Upon impacting the cathodically polarized electrode, the magnetized $\mathrm{Fe}_{3} \mathrm{O}_{4}$ NP is reduced with formation of strongly paramagnetic $\mathrm{Fe}^{2+}$ ions. As the concentration of $\mathrm{Fe}^{2+}$ in the (bulk) electrolyte is zero, these ions diffuse away from the NP 45 surface, allowing a mass transport-controlled dissolution of 
$\mathrm{Fe}_{3} \mathrm{O}_{4}$ at the applied potential. ${ }^{9}$ As in the absence of a magnetic field this diffusion force is the main acting force, dissipation of the ions will be fast and only depending on the $\mathrm{NP}$ radius and the diffusion coefficient of $\mathrm{Fe}^{2+}$ in the 5 respective solution. In contrast, in the presence of a magnetic field gradient, which is particularly strong at the NP surface, where also the $\mathrm{Fe}^{2+}$ concentration is maximum, another force comes into play the magnetic field gradient force

$$
\boldsymbol{f}_{\nabla \mathrm{B}}=\frac{\mu_{0}}{2} c \chi \nabla \boldsymbol{B}^{2}
$$

10 where $\mu_{0}$ denotes the vacuum permeability, $c$ is the concentration and $\chi$ is the molar magnetic susceptibility of the species in solution, $\mathbf{B}$ is the magnetic flux density and $\boldsymbol{\nabla}$ denotes the gradient operator.

Essentially, this force attracts paramagnetic species $(\chi>0)$ 15 to regions of high magnetic gradients, while it has a minor repelling effect on diamagnetic species $(\chi<0)$, as their absolute value is at least one order below that of paramagnetic species.

As the $\mathrm{Fe}^{2+}$ ions formed during the reduction of the $\mathrm{Fe}_{3} \mathrm{O}_{4}$ 20 NPs form high spin octahedral $\mathrm{d}^{6}$ complexes with all potential ligands present in the electrolyte $\left(\mathrm{H}_{2} \mathrm{O}, \mathrm{OH}^{-}, \mathrm{PO}_{4}{ }^{3-}\right),{ }^{17}$ they are strongly paramagnetic $\left(\chi\left(\mathrm{Fe}^{2+}\right) \approx 126 \times 10^{-9} \mathrm{~m}^{3} / \mathrm{mol}\right) .{ }^{18}$ In addition, their release is localized at the NP surface, generating a high concentration of this strongly paramagnetic 25 species exactly in the area of maximum magnetic field gradients. Consequently, a strong attractive force acts on the $\mathrm{Fe}^{2+}$ ions inhibiting their transport away from the $\mathrm{Fe}_{3} \mathrm{O}_{4} \mathrm{NP}$ and therefore significantly slowing down the average current detected per impact spike.

30 To the authors' knowledge this study is the first to observe the action of a magnetic field gradient force acting on single nanoparticles and showing a dramatic effect on the NP's electrochemical dissolution. This nano-scale result is in excellent agreement with previous micro- and macro-scale 35 observations, such as the reduced diffusion of paramagnetic molecules away from ferromagnetic microelectrodes ${ }^{19}$ or the reduced corrosion of magnetized iron wires in areas of high magnetic field gradients. ${ }^{20}$

\section{Conclusions}

${ }_{40}$ Coulometric nanoparticle impact experiments revealed that suspensions containing low concentrations of magnetite NPs show significant magnetic field-induced agglomeration and decreased NP dissolution rates under the influence of a magnetic field. These new insights into the fate of

45 superparamagnetic $\mathrm{Fe}_{3} \mathrm{O}_{4}$ NPs upon application of a magnetic field suggest that this parameter should receive more attention in future chemical and biomedical studies than previously the case. The observed changes in the agglomeration behaviour of individual superparamagnetic particles at concentrations as ${ }_{50}$ low as $\mathrm{ng} / \mathrm{mL}$ are expected to strongly affect their relaxivities and contrasting efficiencies during MRI investigations. ${ }^{21}$ Furthermore, the discovered magnetic field-based change in the NP dissolution behaviour could alter their uptake into the iron metabolism. Thus, their toxicity during or after MRI or 55 hyperthermia treatments might be changed, ${ }^{22}$ which in classic (magnetic field-free) toxicological studies could possibly be misjudged. The observed strong change in mass-transport of paramagnetic ions away from the magnetized particles may also be relevant for magnetic field-assisted micromotor and 60 nanojet applications. ${ }^{23,24}$

\section{Notes and references}

Department of Chemistry, Physical \& Theoretical Chemistry Laboratory, Oxford University, South Parks Road, Oxford, OX1 3QZ, 65 United Kingdom.. Fax: +44(0) 1865 275410; Tel: +44(0) 1865 275957; E-mail: tschulik.kristina@gmail.com , richard.compton@chem.ox.ac.uk

$70 \dagger$ Electronic Supplementary Information (ESI) available: Simulations of the magnetic field distribution near the WE, SEM images and size distributions of the used $\mathrm{Fe}_{3} \mathrm{O}_{4}$ NPs and details of data analysis. See DOI: 10.1039/b000000x/

†Acknowledgements This research was supported by a Marie Curie 75 Intra European Fellowship within the $7^{\text {th }}$ European Community Framework Programme. We thank Kerstin Hennig for SEM sample preparation.

$\lceil$ Footnotes 1 At nano-electrodes limiting (steady-state) currents are established within less than a microsecond, hence we can assume

80 diffusion limitation of the current throughout the spike. ${ }^{11}$ Although here the diffusion is directed away from the NP electrode and not towards it, the same physical description applies.

\section{References}

1. P. Tartaj, M. P. Morales, T. Gonzalez-Carreño, S. VeintemillasVerdaguer, and C. J. Serna, Adv. Mater, 2011, 23, 5243-5249.

2. L. Li, W. Jiang, K. Luo, H. Song, F. Lan, Y. Wu, and Z. Gu, 90 Theranostics, 2013, 3, 595-615.

3. P. B. Santhosh and N. P. Ulrih, Cancer Lett., 2013, 336, 8-17.

4. M. Mahmoudi, H. Hofmann, B. Rothen-Rutishauser, and A. PetriFink, Chem. Rev., 2012, 112, 2323-2338.

5. M. A. Voinov, J. O. S. Pagán, E. Morrison, T. I. Smirnova, and A. I. 95 Smirnov, J. Am. Chem. Soc., 2011, 133, 35-41.

6. Y.-G. Zhou, N. V. Rees, and R. G. Compton, Angew. Chem. Int. Ed., 2011, 50, 4219-4221.

7. G. P. Santos, A. F. A. A. Melo, and F. N. Crespilho, Phys. Chem. Chem. Phys., 2014, 16, 8012.

8. 100 R. E. Rosensweig, Ferrohydrodynamics, Courier Dover Publications, 1997.

9. K. Tschulik, B. Haddou, D. Omanović, N. V. Rees, and R. G. Compton, Nano Res., 2013, 6, 836-841.

10. E. J. F. Dickinson, N. V. Rees, and R. G. Compton, Chem. Phys. Lett., 105 2012, 528, 44-48.

11. J. M. Kahk, N. V. Rees, J. Pillay, R. Tshikhudo, S. Vilakazi, and R. G. Compton, Nano Today, 2012, 7, 174-179.

12. X. Xiao, F.-R. F. Fan, J. Zhou, and A. J. Bard, J. Am. Chem. Soc., 2008, 130, 16669-16677.

13.110 I. Streeter and R. G. Compton, J. Phys. Chem. C, 2007, 111, 1804918054.

14. T. Z. Fahidy, J. Appl. Electrochem., 1983, 13, 553-563.

15. G. Hinds, J. M. D. Coey, and M. E. G. Lyons, Electrochem. Commun., 2001, 3, 215-218.

16.115 D. R. Lide, CRC handbook of chemistry and physics, Taylor \& Francis Group, 89th edn., 2008.

17. Y. Shimura, Bull. Chem. Soc. Jpn., 1988, 61, 693-698.

18. J. M. D. Coey, F. M. F. Rhen, P. Dunne, and S. McMurry, J. Solid State Electrochem., 2007, 11, 711-717.

19.120 K. M. Grant, J. W. Hemmert, and H. S. White, Electrochem. Commun., 1999, 1, 319-323.

20. R. Sueptitz, K. Tschulik, M. Uhlemann, A. Gebert, and L. Schultz, 
Electrochimica Acta, 2010, 55, 5200-5203.

21. H. Amiri, L. Bordonali, A. Lascialfari, S. Wan, M. P. Monopoli, I. Lynch, S. Laurent, and M. Mahmoudi, Nanoscale, 2013, 5, 8656.

22. S. Puntarulo, Mol. Aspects Med., 2005, 26, 299-312.

23. 5 G. Zhao and M. Pumera, Langmuir, 2013, 29, 7411-7415.

24. G. Zhao, S. Sanchez, O. G. Schmidt, and M. Pumera, Chem Commun., 2012, 48, 10090-10092. 